\section{Acute erythroid leukemias have a distinct molecular hierarchy from non-erythroid acute myeloid leukemias}

In 2016 the World Health Organization (WHO) has reclassified the acute erythroid/myeloid type of acute myeloid leukemia (AML) (thereafter designated M6AML), formerly described as M6a-AML by the FrenchAmerican-British cooperative group, in either AML or myelodysplastic syndrome (MDS) depending on the number of blasts. ${ }^{1,2} \mathrm{We}$ previously proposed a molecular classification of M6-AML in adult patients using targeted next generation sequencing (tNGS) and array comparative genomic hybridization $(\mathrm{aCGH}) .{ }^{3}$ We showed that the adult M6-AML share the same molecular profile as the other AML and were mainly distributed in four major classes with mutations in either NPM1, transcription factors (e.g. RUNX1), splicing factors and/or chromatin modifiers (e.g. ASXL1, SRSF2, U2AF1), or TP53. ${ }^{3}$ This was confirmed and refined in a recent, more comprehensive study. ${ }^{4}$ Based on these results and on the 2016 WHO classification we revisited the gene mutations and prognosis of our series of M6-AML. ${ }^{5}$ No molecular differences in the number of mutations or in the molecular classes were found between the M6-AML regardless of whether they were reclassified as AML or as myelodysplastic syndromes (AEL-MDS). ${ }^{5}$ Thus, in our series, the WHO 2016 had no impact on the prognosis whereas molecular stratification did. ${ }^{5}$ M6-AML seem to be AML in their own right: although they may have some particularities such as frequent TP53 bi-allelic alteration ${ }^{6}$ or rare gene fusions, ${ }^{4}$ globally they do not have specific molecular profiles and their prognosis is similar or close to non-M6AML and better assessed by mutations. This led us to surmise that an important part of the difference between M6-AML and non-M6-AML could be due to a different leukemic cell-of-origin.

To test this hypothesis, we compared the mutations present in different compartments of the cellular hierarchy of seven M6-AML and five non-M6-AML. The 12 patients were selected according to their whole bone marrow mutational status and to the availability of live cells in our biobank. In the M6-AML group, three NPM1-mutated patients, three TP53-mutated patients and one patient mutated in U2AF1 (splicing factors and/or chromatin modifiers class) were studied. The nonM6-AML group comprised three NPM1-mutated and two TP53-mutated patients. The main clinical, biological and molecular characteristics are presented in the Online Supplementary Table S1. We used multi-parameter-fluorescence-activated cell sorting (FACS) to isolate the different cell compartments of the hematopoietic hierarchy and Sanger-sequencing to establish the molecular status of each isolated subpopulation.

After obtaining the written consent of the patients according to our ethical committee regulations and biobank procedures, we did a $\mathrm{CD} 34^{+}$enrichment from the 12 bone marrow samples collected at diagnosis and obtained from the IPC/CRCM biobank, which operates under authorization \# AC-2007-33 granted by the French Ministry of Research. Cell separation was done by using immunomagnetic CD34 beads from Milteny Biotec. To isolate the different immature subpopulations according to the classical hierarchical model of hematopoiesis, $\mathrm{CD} 34^{+}$cells were subsequently costained with a mixture of eight monoclonal antibodies and then sorted using a FACSARIAIII (BD Biosciences). In parallel, the CD34 population was costained with a mixture of seven monoclonal antibodies. Cell sorting is detailed in the Online

Table 1. Clinical and molecular features of cell subpopulations of the hematopoietic hierarchy of seven erythroid acute myeloid leukemias (M6-AML) (3 NPM1-, 1 U2AF1- and 3 TP53-mutated) and five non-M6-AML (3 NPM1- and 2 TP53-mutated).

\begin{tabular}{|c|c|c|c|c|c|c|c|c|c|c|c|c|c|c|c|c|c|c|}
\hline & & & & & & \multicolumn{8}{|c|}{ CD34+ CEUS } & \multicolumn{5}{|c|}{ CD34-CELLS } \\
\hline & & $\begin{array}{c}\text { Classification } \\
\text { FAB }\end{array}$ & $\begin{array}{l}\text { Classiffication WHO } \\
2016\end{array}$ & $\begin{array}{c}\text { Sequencing } \\
\text { (Sanger) }\end{array}$ & VAF (NGS) & HSC & Lsc & MPP & LMPP & CMP & GMP & ap & MEP & C0235 & c033 & CD16 & CD19 & $\mathrm{CO} 3$ \\
\hline & \multirow{2}{*}{ HD-2295 } & \multirow{2}{*}{ AML-MG } & \multirow{2}{*}{ MDS-EB-2 } & NPM1 & 35,2 & & & & & & & & & & & & & \\
\hline & & & & N-RAS & 8,4 & & & & & & & & & & & & & \\
\hline & HO-2256 & AML-M6 & MDS-EB-2 & NPMI & 22,2 & & & & & & & & & & & & & \\
\hline & \multirow{2}{*}{ HD-2271 } & \multirow{2}{*}{ AML-M6 } & \multirow{2}{*}{ AML-MRC } & NPM1 & 29,6 & & & & & & & & & & & & & \\
\hline & & & & N-RAS & 31,3 & & & & & & & & & & & & & \\
\hline & \multirow{2}{*}{ HD-2180 } & \multirow{2}{*}{ AML-ME } & \multirow{2}{*}{ MOS-EB-2 } & U2AF1 & 14 & & & & & & & & & & & & & \\
\hline & & & & TRIM1O & 25,9 & & & & & & & & & & & & & \\
\hline & HO-2170 & AML-M6 & MOS-EB-2 & TP53* & $32.4 / 30.1$ & & & & & & & & & & & & & \\
\hline & HO-2322 & AML-M6 & AML-MRC & TP53 & 83,3 & & & & & & & & & & & & & \\
\hline & \multirow{2}{*}{ HD-2622 } & \multirow{2}{*}{ AML-M6 } & \multirow{2}{*}{ AML-MRC } & TPS3* & $31.99 / 23.89$ & & & & & & & & & & & & & \\
\hline & & & & оммтза & 30,21 & & & & & & & & & & & & & \\
\hline \multirow{2}{*}{$\begin{array}{l}\text { Mutated } \\
\text { Wild-type }\end{array}$} & $\mathrm{HB}-1147$ & AML-M4 & AML & NPMI & 20 & & & & & & & & & & & & & \\
\hline & \multirow{2}{*}{ HB-1289 } & \multirow{2}{*}{ AML-M4 } & \multirow{2}{*}{ AML } & NPM1 & 30,3 & & & & & & & & & & & & & \\
\hline No data due to & & & & ONMTSA & 44,13 & & & & & & & & & & & & & \\
\hline failure & HO-2683 & AML-M1 & AML & NPM1 & nd & & & & & & & & & & & & & \\
\hline \multirow{3}{*}{$\begin{array}{c}\text { loss of } \\
\text { heterozygosity }\end{array}$} & \multirow{2}{*}{ HB-1007 } & \multirow{2}{*}{ AML-M1 } & \multirow{2}{*}{ AML } & TPS3 & 86,58 & & & & & & & & & & & & & \\
\hline & & & & TET2 & 77,9 & & & & & & & & & & & & & \\
\hline & $\mathrm{HB}-1317$ & AML-M4 & AML & TP53 & 71,87 & & & & & & & & & & & & & \\
\hline
\end{tabular}

TP53*: double mutation; M6-AML: erythroid acute myeloid leukemia; AML: acute myeloid leukemia; VAF: variant allele frequency; HSC: hematopoietic stem cell; LSC: leukemic stem cell; MPP: multipotent progenitor; LMPP: lymphoid-myeloid pluripotent progenitor; CMP: common myeloid progenitor; CLP: common lymphoid progenitor; MEP: megakaryocyte erythroid progenitor; GMP: granulocyte-monocyte progenitor. 
Supplementary Materials and Methods and an example of our analyses is shown in the Online Supplementary Figure $S 1$ and the proportion of $\mathrm{CD} 34^{+}$and $\mathrm{CD} 34^{-}$subpopulations obtained in each case is shown in the Online Supplementary Figure S2. The subpopulations obtained were then Sanger-sequenced for one or two respective driver gene(s). Some were also studied by aCGH when possible, as previously described.,

Results are presented in Figure 1 and Table 1. The mutations in the studied driver genes were present in various compartments of the seven M6-AML and the five non-M6-AML. The main difference was that mutations in the M6-AML affected the red cell lineage (MEP and $\mathrm{CD} 235^{+}$) but did not affect CD235 cells in the non-M6AML. Alterations detected by aCGH (e.g. loss of chromosome 7 , chromosome arm $17 p$ or within the $5 q$ arm) followed the same pattern (Online Supplementary Figure S3). Thus, the presence of driver alterations in the red cell lineage, in particular in $\mathrm{CD}_{2} 35^{+}$, seems to be a feature of
M6-AML as compared to non-M6-AML.

How can this difference be explained? Several mechanisms, genetic and/or epigenetic, and cell-autonomous and/or non-cell autonomous, may account for differences in phenotype. One possibility is that the lineage potency of the targeted progenitor cell in M6-AML includes erythropoiesis. Another possibility is that the presence of driver mutations in non-M6-AML is deleterious for the proper development and function of the red cell lineage and that additional mutations in M6-AML counter this effect and allow the red cell lineage to be a component of the leukemia. To get further insight we studied our published series of $58 \mathrm{M} 6-\mathrm{AML}^{3,5}$ by tNGS of a panel of 227 genes known to play a role in leukemogenesis and/or erythropoiesis (Online Supplementary Table S2). We found three truncating mutations, two in exon 8 of the EPOR gene (S407X and L436fs) in cases respectively mutated in TP53 and RUNX1 driver genes, and one in exon 7 of the TRIM10 gene (G377fs) in the U2AF1-mutated case

\section{A NPM1 mutation: 3 M6-AMLs and 3 non-M6-AML}
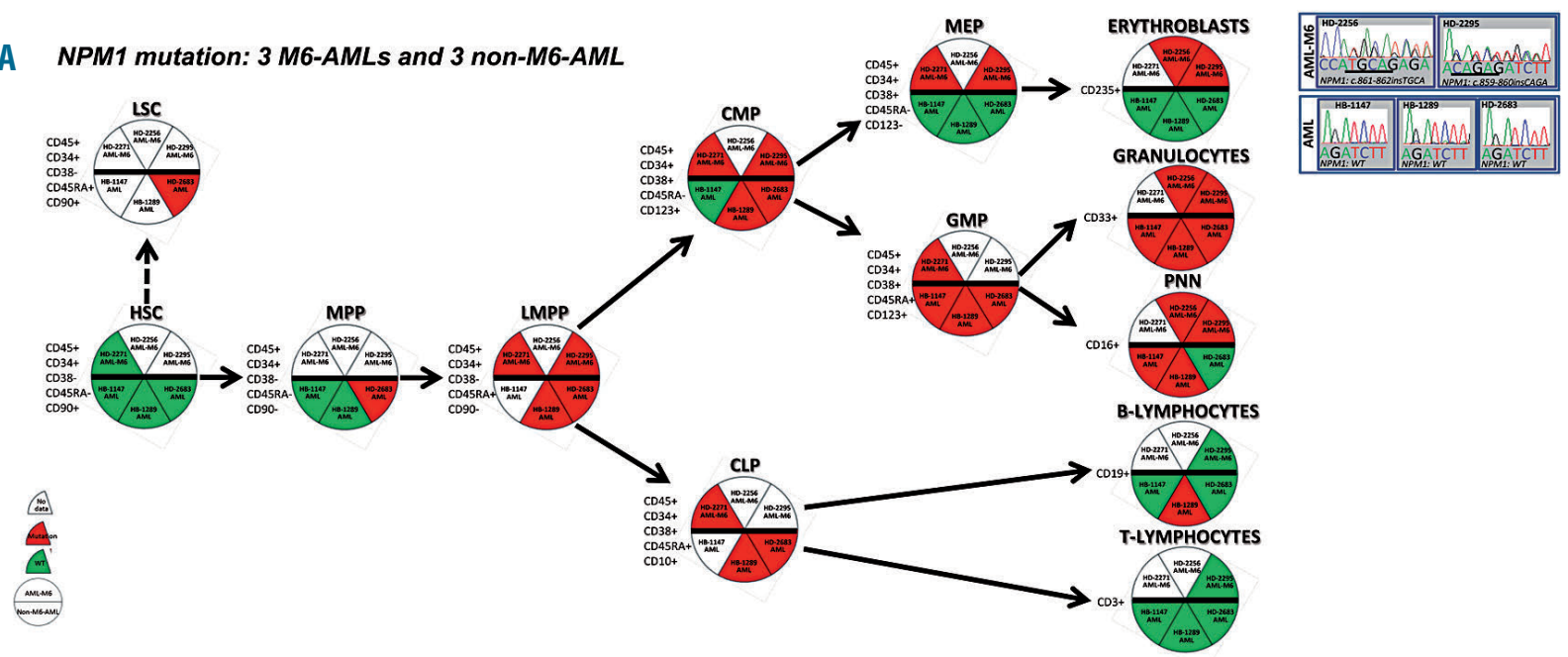

B TP53 mutation: 3 M6-AMLs and 2 non-M6-AML

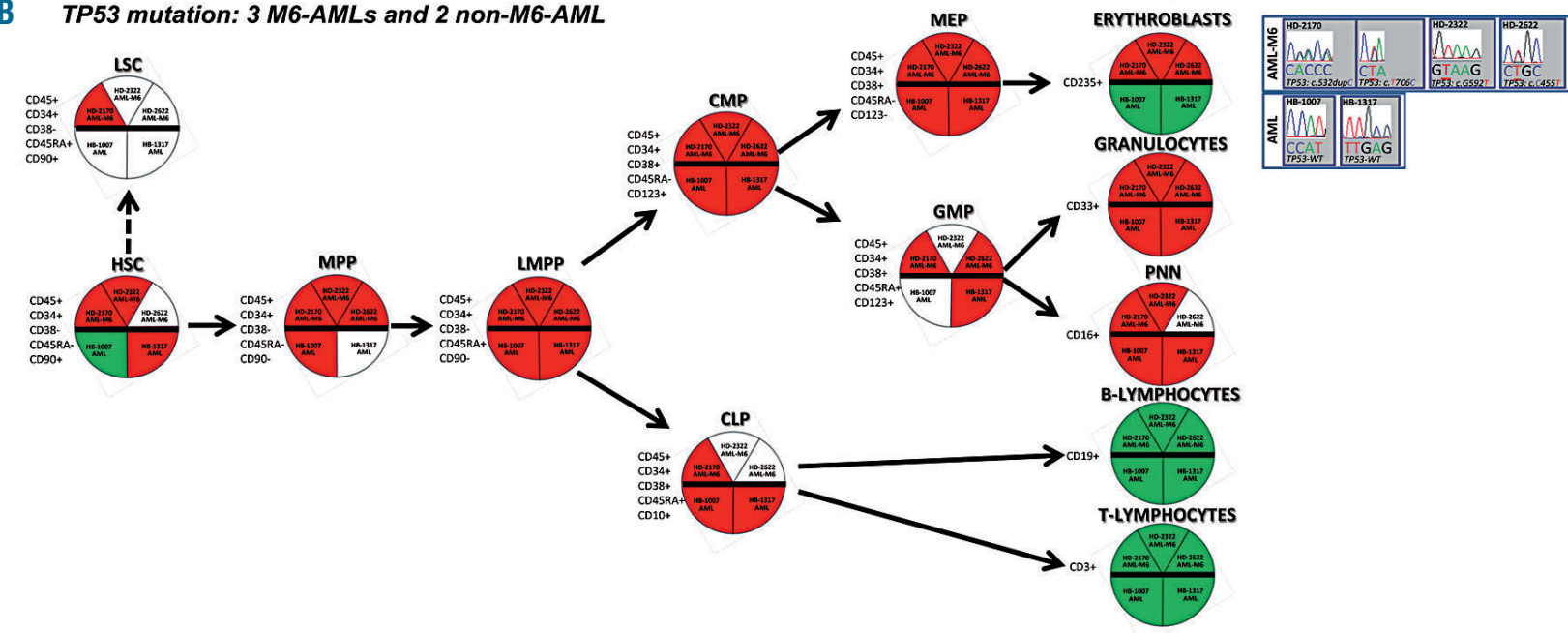

Figure 1. Distribution of NPM1 and TP53 mutations in hematopoietic compartments of erythroid acute myeloid leukemia (M6-AML) and non-M6-AML. (A) Three M6-AML and three non-M6-AML sequenced for NPM1. Each circle represents a cell at a different level of the classical hematopoietic hierarchy from the most immature to mature population. The immunophenotype of these cells corresponds to the cell sorting performed. Each cell/circle is separated in two by a bold black line with M6-AML above and non-M6-AML below. Inside each cell/circle, each slice corresponds to a Sanger-sequenced case with NPM1 mutation: red means mutated, green non-mutated and white no data. Results of NPM1 sequencing in erythroblasts are indicated to the right. M6-ALM: erythroid acute myeloid leukemia; AML: acute myeloid leukemia; HSC: hematopoietic stem cell, LSC: leukemic stem cell; MPP: multipotent progenitor; LMPP: lymphoid-myeloid pluripotent progenitor; CMP: common myeloid progenitor; CLP: common lymphoid progenitor; MEP: megakaryocyte erythroid progenitor; GMP: granulocyte-monocyte progenitor. (B) Three M6-AML and two non-M6-AML sequenced for TP53. Symbols as in (A). 
(Online Supplementary Figure S4). EPOR exon 8 truncating mutations are activating in congenital erythrocytosis. ${ }^{10}$ Unfortunately, the lack of availability of live cells prevented us to look for the EPOR mutations in the cell compartments of these cases. However, the mutation in TRIM10 could be verified by Sanger-sequencing of the subpopulations: the frameshift mutation was present in the CD235 ${ }^{+}$cells (Table 1 ). TRIM10/HERF1, a member of the tripartite motif (TRIM) family, is a zinc finger protein that shares similarities with PML and TRIM27, and is involved in the biology of the red cell lineage. ${ }^{11}$ Its inhibition induces a block in the differentiation of erythroid cells. The mutation would lead to a protein truncated of its C-terminal part, as does for PML the fusion with RARA in M3-AML, and for TRIM27 the fusion with the RET tyrosine kinase receptor in thyroid carcinomas. These data suggest a cooperation of a driver gene and a "red cell gene" resulting in the alteration of erythroid proliferation and differentiation observed in M6-AML. Other alterations, such as GATA2 or gene fusions may play a similar role. ${ }^{4,12}$ Some mutations present only in a subpopulation of cells, and consequently harboring a low variant allele frequency (VAF) in the whole sample, may be missed in low-depth sequencing analyses.

These preliminary observations need to be confirmed and extended to understand the physiopathology of M6AML. As such, they suggest that in M6-AML, mutations occur in a progenitor that has a potency or fate different from that of the non-M6-AML progenitor. In M6-AML but not in non-M6-AML, they would target a progenitor able to differentiate along both the granulocytic lineage and the red cell lineage, inducing the specific phenotype of M6-AML. Some of these mutations seem to occur in genes involved in the red cell lineage; because they are expressed in this lineage, the mutations would be fixed and would explain a dominant abnormal proliferation.

Nathalie Cervera, ${ }^{1}$ Anne-Catherine Lhoumeau, ${ }^{1,2}$ José Adélaïde, Arnaud Guille, Anne Murati, ${ }^{1,2}$ Marie-Joëlle Mozziconacci, ${ }^{2}$ Norbert Vey, ${ }^{3}$ Daniel Birnbaum ${ }^{1}$ and Véronique Gelsi-Boyer,

'Laboratoire d'Oncologie Prédictive, Centre de Recherche en Cancérologie de Marseille UMR1068 Inserm, Institut Paoli-Calmettes, CNRS UMR7258, Aix-Marseille Université UM105, Marseille; ¿Département de BioPathologie, Institut Paoli-Calmettes, Marseille and ${ }^{3}$ Département d'Hématologie, Institut Paoli-Calmettes, Marseille, France
Acknowledgements: we thank the flow cytometric platform, and the biobank and C. Chabannon for the samples.

Funding: our work is supported by Inserm, the Paoli-Calmettes Institute and a SIRIC grant (INCa-DGOS-Inserm 6038, specific grant to VGB).

Correspondence:

VERONIQUE GELSI-BOYER - gelsiv@ipc.unicancer.fr

doi:10.3324/haematol.2019.231142

Information on authorship, contributions, and financial \& other disclosures was provided by the authors and is available with the online version of this article at www. haematologica.org.

\section{References}

1. Arber DA, Orazi A, Hasserian R, et al. The 2016 revision to the World Health Organization classification of myeloid neoplasms and acute leukemia. Blood. 2016;127(20):2391-2405.

2. Bennett JM, Catovsky D, Daniel MT, et al. Proposed revised criteria for the classification of acute myeloid leukemia: a report of the French-American-British Cooperative Group. Ann Intern Med. 1985;103(4):620-625.

3. Cervera N, Carbuccia N, Garnier S, et al. Molecular characterization of acute erythroid leukemia (M6-AML) using targeted next-generation sequencing [letter]. Leukemia. 2016;30(4):966-970.

4. Iacobucci I, Wen J, Meggendorfer M, et al. Genomic subtyping and therapeutic targeting of acute erythroleukemia. Nat Genet. 2019;51(4):694-704

5. Cervera, N, Carbuccia N, Mozziconacci MJ, et al. Revisiting gene mutations and prognosis of ex-M6a-acute erythroid leukemia with regard to the new WHO classification [letter]. Blood Cancer J. 2017;7(8):e594.

6. Montalban-Bravo G, Benton CB, Wang SA, et al. More than 1 TP53 abnormality is a dominant characteristic of pure erythroid leukemia. Blood. 2017;129(18):2584-2587.

7. Dalerba P, Cho RW, Clarke MF. Cancer stem cells: models and concepts. Annu Rev Med.2007;58:267-284.

8. Etienne A, Carbuccia N, Adélaïde J, et al. Rearrangements involving $12 \mathrm{q}$ in myeloproliferative disorders: possible role of HMGA2 and SOCS2 genes. Cancer Genet. Cytogenet. 2007;176(1):80-88.

9. Adélaide J, Finetti P, Bekhouche I, et al. Integrated profiling of basal and luminal breast cancers. Cancer Res. 2007;67(24):11565-11575.

10. Huang, LJ, Shen, YM, Bulut, GB. Advances in understanding the pathogenesis of primary familial and congenital polycythaemia. Br J Haematol. 2010;148(6):844-852

11. Harada H, Harada Y, O'Brien DP, Rice DS, Naeve CW, Downing JR. HERF1, a novel hematopoiesis-specific RING finger protein, is required for terminal differentiation of erythroid cells. Mol Cell Biol. 1999;19(5):3808-3815

12. Ping $\mathrm{N}$, Sun $\mathrm{A}$, Song $\mathrm{Y}$, et al. Exome sequencing identifies highly recurrent somatic GATA2 and CEBPA mutations in acute erythroid leukemia. Leukemia. 2017;31(1):195-202. 